\title{
Proposal of a Medical Algorithm Based on the Application of Digital Image Processing and Visual Communication Techniques
}

Ana Carolina Borges Monteiro

$$
\text { Yuzo Iano }
$$

Reinaldo Padilha França 


\title{
Proposal of a Medical Algorithm Based on the Application of Digital Image Processing and Visual Communication Techniques
}

\author{
Ana Carolina Borges Monteiro, Yuzo Iano, and Reinaldo Padilha França
}

\begin{abstract}
Blood tests help detect various types of diseases, where the erythrocytes count (RBC) and leukocytes (WBC) can use automatic and/or manual methodologies. The evaluation of RBC and WBC has a direct impact on the diagnosis of anemia, leukemia, viral, parasitic and viral infections. Given the importance and wide applicability of the Watershed Transform (WT) and Morphological Operations (MO), they can segment medical images to increase the efficiency and reliability of medical diagnostics. This study uses a WT-MO algorithm for segmentation, detection, and counting of blood cells that attend the criteria of efficiency and reliability. This methodology may be the first step in making blood tests more accessible to people from developing and underdeveloped countries. The WT-MO algorithm has been benchmarked using 30 microscopy images of a blood smear. The WT-MO algorithm results presented high accuracy $(93 \%)$. The simulations of the algorithm executed in different hardware platforms presented average simulation and processing time with less than 3 seconds per sample. Therefore, the WT-MO based algorithm is accurate, reliable and a low-cost technique, which can be applied as a third methodology to perform the laboratory tests and to speed up further medical diagnostic.
\end{abstract}

Keywords - Image segmentation, Watershed Transform, Blood cells, Image processing, Biomedical engineering, Soft computing.

\section{INTRODUCTION}

\section{A. Image Segmentation}

Often, image segmentation is the first step in

A.C.B. Monteiro is pursuing an M.Sc. degree in Electrical Eng. (EE), at the Lab. of Vis. Comm. (LCV) at the State Univ. of Campinas (UNICAMP), monteiro@decom.fee.unicamp.br

Dr. Y. Iano is the LCV-UNICAMP head, yuzo@decom.fee.unicamp.br

R. Padilha is currently pursuing an M.Sc. degree in EE at LCVUNICAMP, padilha@decom.fee.unicamp.br general object recognition in many applications for identifying regions of interest (ROIs) in a scene or annotating the data [1] [2].

Image segmentation is an essential process for the image analysis task. The segmentation process consists in the act of fractionating an image into groups of spatially connected pixels homogeneously [3] [4].

There are many techniques for describing and recognizing correct image viewing, with image comprehension being a highly process dependent on the outcome of segmentation processing [3] [4]. There are many methodologies of image segmentation. However, the focus of this study is the Watershed Transform (WT).

The intuitive idea of the Watershed Transform comes from the geography: landscape of topography reliefs responsible for the formation of watersheds, these being divided into lines of domain aiming the attraction of rainwater over the region. An alternative approach is to imagine a landscape being immersed in a lake, with holes drilled in local minimums [5] [6].

In this way, the river basins fill with water starting at the minimum place, where the water meets barriers. When the water level is at the highest peak in the landscape the process is stopped. As a result, the landscape is fractioned into regions or basins separated by dams called basin hydrography lines. The image segmentation process presents wide applicability, being used to solve problems in several areas [5] [6].

There are many researchers about still image and video segmentation. These studies are wide and includes many techniques as motion-based segmentation [7][8][9][10], spatial segmentation and motion tracking [11][12][43][44], moving objects extraction [13][14][43][44], and region growing using spatio-temporal similarity [15][16]. Many of the existing segmentation techniques, such as direct clustering methods in color space [17][18], work well on homogeneous color regions but natural scenes are rich in color 
and texture. The existence of texture and motion can also be used to characterize pathologies.

The algorithms for image segmentation based on the WT [19] need a way of measuring the similarity or difference between pixels that form an image. So, the WT needs to be combined with some gradient of an image complex in scenes. One example of this is the MPEG-4 standard that needs segmentation to object-based video coding.

It is possible to associate different segmentation techniques to solve these image segmentation problems. The combination of morphological segmentation (MO) and the WT technique has been applied to improve sports scenes. This process consisted of coarse segmentation by binary reconstruction based on the areas detected by thresholding the color histogram and segmentation by WT. This process results in segmentation image sequences in sports scenes containing brisk movement. That is an important feature in automatic morphological segmentation during broadcast [20].

Other studies showed that the WT works quite well for image segmentation. The WT technique computes the dissimilarity of pixels using RGB-D cues and gradient maps, where an RGB-D image is a combination of an RGB image and the corresponding image depth map. The process captures an image gradient map to enhance and explain directional variations in image intensities. A distance function or metric measures the similarity between adjacent pixels according to RGB-D values [21].

Due to the importance and wide applicability of the WT in image segmentation regardless of the dimensionality of the image space, and applying the essence of the multidisciplinarity of scientific research. So, the application of the WT in biomedical engineering can help the development of better blood diagnosis algorithms.

\section{B. Blood Cells}

The human blood consists of approximately $55 \%$ plasma and $45 \%$ blood cells. The blood cells that compose the blood are erythrocytes, leukocytes, and platelets [22] [23]. The cells are responsible for the homeostasis of the organism (through the transport of gases), organism defense against distinct etiological agents, tissue repair, and blood coagulation. However, such observations could only occur through the invention of the optical microscope [23].
Through observations in optical microscopy, biconvex disc-shaped structures with no cell nucleus were visualized. These cells were called erythrocytes. Its main function is the transport of gases by the organism [24] [25].

Leukocytes are the defense cells of the organism, being morphologically characterized by the presence of segmented nuclei in lobes and the presence of granules [26]. The leukocytes are divided into 5 cell subtypes: neutrophils, basophils, eosinophils, monocytes and lymphocytes. These cells present morphological differences according to the function performed such as the defense of the organism against external agents such as bacteria, parasites, and viruses [27] [28].

Over time, the study of blood cells was restricted to just the observation of cell morphology began to use specific methods. However, new technologies do not negate the need for conventional microscopy. This new methodology responsible for the analysis of blood cells was called hemogram [28] [29].

The hemogram is a laboratory exam for the quantification and qualification of blood cells [8]. In this exam the erythrocytes should be expressed between 4.0 to $6.0 \times 106 / \mathrm{mm}^{3}$ of blood. Values lower or higher than this parameter is indicative of genetic anemias or deficit of food, leukemias or polycythemias [30].

Commonly, the total leukocyte count should be between 3.500 and 10.000 leukocytes $/ \mathrm{mm}^{3}$ of blood. Changes in this value may be indicative of leukemias, sedentary lifestyle, skin infections, stress, bacterial infections, HIV, among others [27] [28] [30].

Today's importance of hemograms demands new technology from the field of biomedical engineering [31]. The MATLAB ${ }^{\odot}$ software contains several toolboxes with algorithms that have broad applicability such as the Watershed Transform, and the Morphological Operations (MOs). Initially, this work segments images by labeling pixels, and detecting objects corresponding to specific stain patterns [32] with the objective of identifying blood cells based on their morphological characteristics. Hence, this algorithm may automate the count of blood cells.

This work is organized as follows. Section II discusses the traditional methodology. Section III describes the proposed method. Experimental results appear in Section IV. This manuscript concludes with Section V. 


\section{TRADITIONAL METHODOLOGY}

The quantification and qualification of the blood cells are performed by an examination called hemogram. This exam is currently performed through two methodologies: manual and automatic [29].

The manual hemogram depends entirely on human performance, and it uses non-automated equipment relying on either a blood smear sample or a hemocytometer using a standard optical microscope. This work handles the manual blood count from a blood smear to determine the number of blood cells, where the blood smear was obtained by depositing a few microliters of blood on a glass slide. The monolayer regions are mechanically scanned for counting the total number of cells.

The specimen can either be seen directly over the microscope or from image files. Even though the manual technique is more laborious and timeconsuming, it allows the use of several objective lenses for the standard microscope for highquality, inexpensive, and careful visual examination of the samples.

This methodology presents a lower cost but requires more time for the release of reports. Besides, it gives less reliability because it depends on manual counts and calculations performed by health professionals. It is considered a good alternative for small laboratories, where the demand for exams is low, and the cost with the acquisition with hematological equipment and reagents does not match the cost-benefit of the process [29]. A common shortcoming of the manual counting method is its error due to the way the specimen is observed. A standard microscope performance is influenced by its space-bandwidth product, leading to a trade-off between the image resolution and the microscope Field-of-View (FOV). Generally, to view and count blood cells under a conventional microscope, an objective with an adequate magnification. However, in manual methods, the FOV is very small, which necessitates mechanical scanning of the specimen during the counting process. This setting is unfavorable because the scanning movement has to be correctly aligned and regulated to bypass any overlap of the regions undergoing scanning. Another disadvantage is the physical strain on the professionals performing the manual scans by direct observation of the microscope, which may be detrimental to the physician diagnostic.

The automation of the hemogram implies greater agility in performing the exams and in the release of reports. However, it is a more expensive methodology when compared to the manual methodology. Automation is based on impedance and flow cytometry methods for the differentiation and counting of blood cells [6][28] [33].

However, before acquiring a hematological equipment's it is necessary to take into consideration the following parameters: automation equipment's versus the type of patient attended; demand for daily exams, cost of each exam, interfacing and training of employees [6][29].

It is important to emphasize that even with the acquisition of hematological equipment, the manual hemogram is not a dispensed practice. Manual hematology is a recommended technique for confirmation of hematological findings, such as pediatric patients, patients over 75 years of age, cancer patients, patients with suspected leukemia or polycythemia, patients with leukocytosis and patients with severe state of hospitalization in Intensive Care Unit (ICU) [29].

\section{WATERSHED TRANSFORM WITH MORFOLOGICAL OPERATIONS (WT-MOS)}

The experiments were conducted through digital images acquired by the camera coupled to the optical microscope to obtain images of blood smears. The fields of the blood smear contained erythrocytes and leukocytes in different sizes, colors, and quantities.

The system modeling, as well as the program, were implemented using an IDE the MATLAB $\mathbb{}$ version 2014a. In this environment, image segmentation algorithms were developed for counting and detection of erythrocytes and leukocytes.

The algorithm developed has as an emphasis the image segmentation process through of the union of WT techniques and morphological operations. The detection and counting of erythrocytes were performed through the WT, while the counted leukocytes were the result of the morphological operations process.

The counting and differentiation processes of these cells are performed based on the morphological characteristics of the blood cells. The logic of the algorithm is shown in Figure 1. 


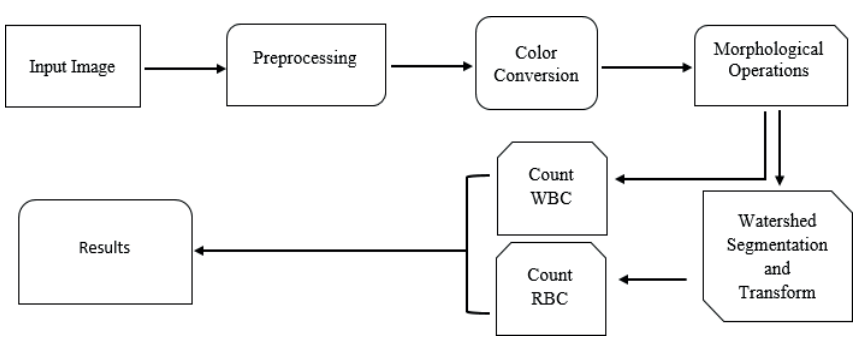

Figure 1 Block diagram for the WT-MO algorithm

\section{A. Erythrocytes Counts}

The segmentation process consists in the act of fractionating an image into groups of spatially connected pixels homogeneously [34][35]. Image segmentation can be used alternatively as a pixel labeling process, where all pixels belonging to the same homogeneous region are marked with the same label, as shown in Figure 2 [35] [36].

The definition of homogeneity of a region of the image presents a particular concept of the segmentation process because each image presents its pattern of pixels [32] [37].
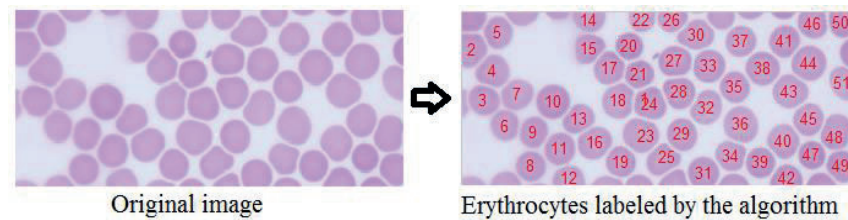

Figure 2 Erithrocytes labeled by the Watershed Transform

For the labeling of erythrocytes, the pixel is assumed as a variable directly related to the morphological characteristics of the cells under analysis. In that case, the labeling would consider the size and staining of erythrocytes [32].

\section{Leukocytes Counts}

Morphological Operations (MOs) constitute a broad set of image processing operations based on the shape of binary images. The morphological operations are applied to the structural element at the input of an image creating an output of the same size, as shown in Figure 3 [36] [37].

In this way, the value of the pixels in the image output is based on the comparison of pixels corresponding to the pixels of the image input and neighboring pixels. The number of pixels added or removed from objects in an image depends on the size and format of the structural element used to process the image. Morphological operations consist of the following steps: dilatation; erosion; opening; closure and reconstruction [36][37]. The dilation consists of adding pixels to the edges of objects in an image to the purpose of repairing breaks or possible intruding elements in the image. Erosion, in turn, consists of the removal of pixels from the edges of the image, being used to divide bound objects or remove extrusions. The aperture is applied for the elimination of protrusions and/or smooth contours [19]. In turn, the closure is used to merge fine and long gaps and gaps, eliminate small holes, and fill spaces in the contour and smooth out the contours. The reconstruction is responsible for extracting relevant information referring to the shapes present in the image [19].

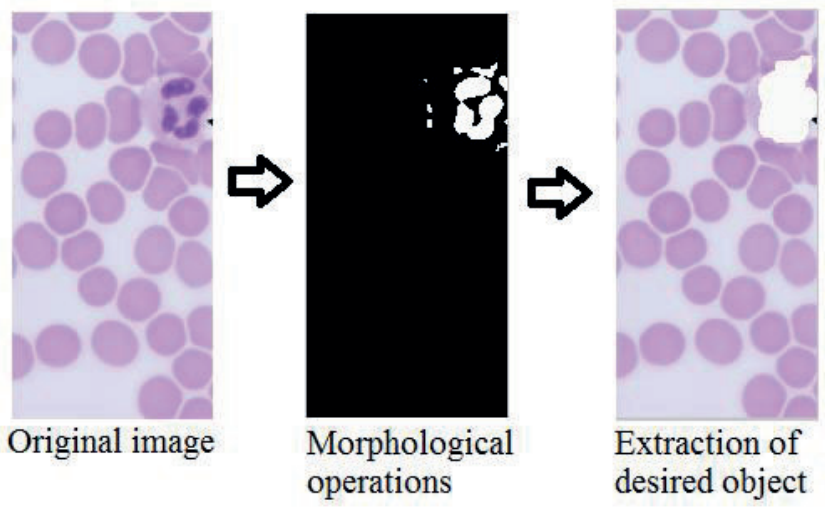

Figure 3 Leukocyte count by the WT-MO algorithm

\section{RESULTS}

Thirty microscopy images containing a total of 6453 erythrocytes and 187 leukocytes served as benchmarks. Both cell types were automatically and manually counted, with subsequent counting by the algorithms of detection and counting of blood cells. Subsequently, the results were compared to determine the accuracy of the WTMO approach, according to Figures 4 and 5.

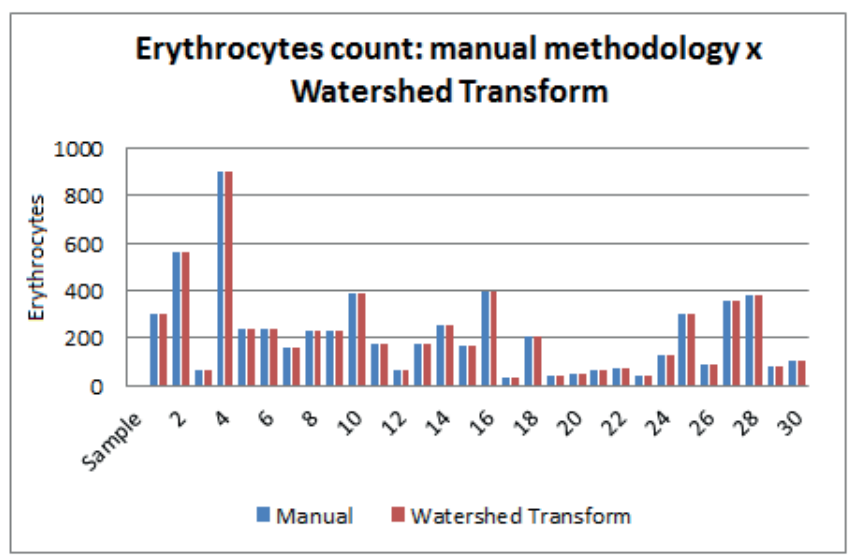

Figure 4 Comparison of the erythrocyte counts by the manual method with the automated counting using the 
Watershed Transform

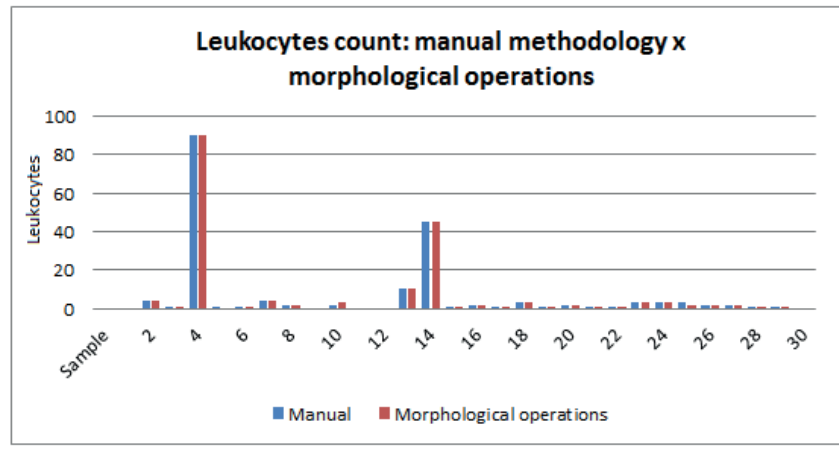

Figure 5 Comparison of the leukocyte counts by the manual method with the automated counting using morphological operations process

The comparison of the manual methodology of cell counting with the developed algorithm demonstrated an accuracy of $93 \%$ in the detection of erythrocytes and $90 \%$ in the detection of leukocytes.

The developed procedure was also submitted to evaluations of runtime and execution time. For this, executions of each command were realized in machines with different hardware configurations, consisting of a Dual Core processor, with $2 \mathrm{~GB}$ of RAM, an Intel Core i3 processor, with $4 \mathrm{~GB}$ of RAM and an Intel Core i5 processor with $8 \mathrm{~GB}$ RAM.

The tictoc MATLABC command measured the time spent by each of the algorithms during their execution (in seconds). The algorithm also used the cputime command, whose function is to return the total CPU time (in seconds) since the moment it started [38] [39] [40].

The tictoc function measures the algorithm execution time. The cputime function calculates its computational time [39] [40]. Figures $6,7,8,9,10$ and 11 present the performance and efficiency of the algorithm.

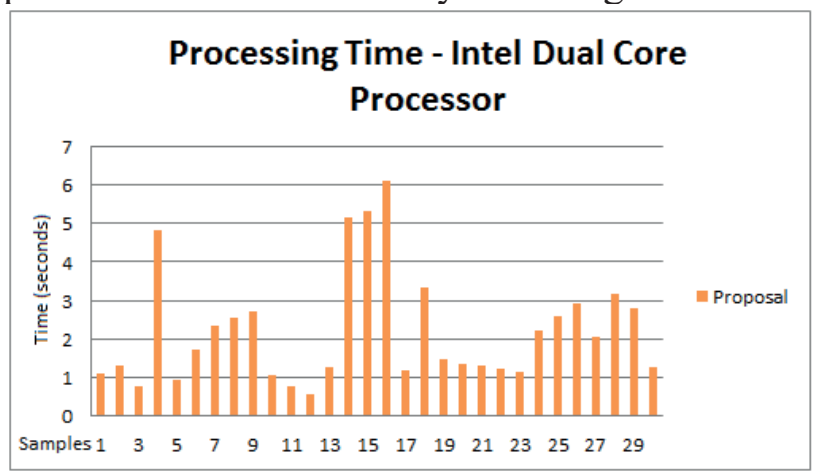

Figure 6 Processing times in a Dual Core processor

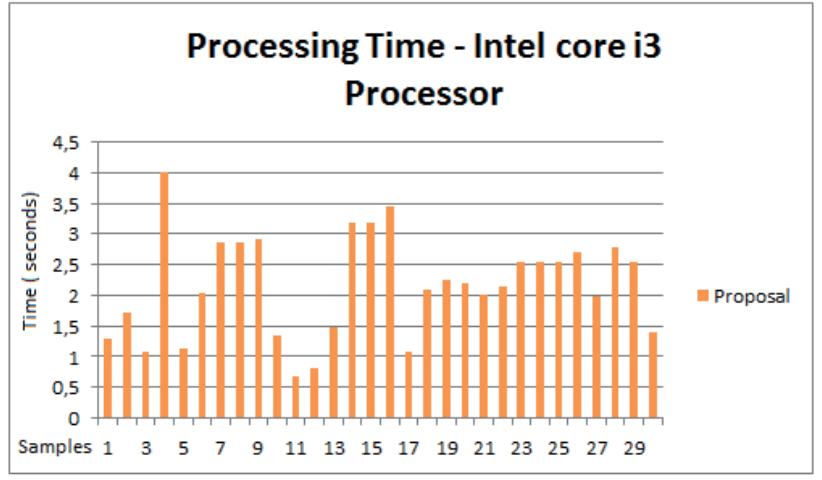

Figure 7 Processing time in Intel Core i3 processor

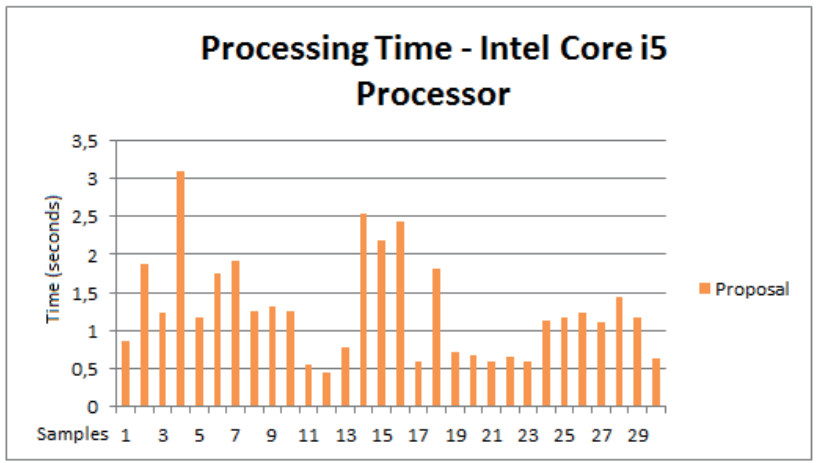

Figure 8 Processing times in Intel Core i5 processor

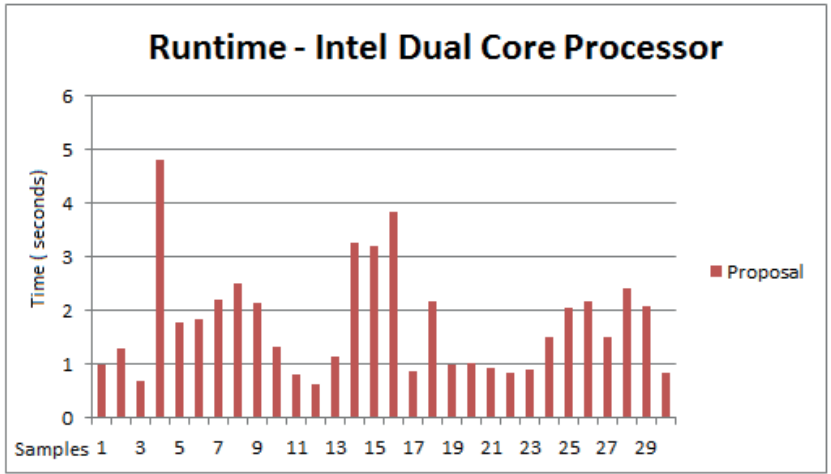

Figure 9 Execution times for the Dual Core processor

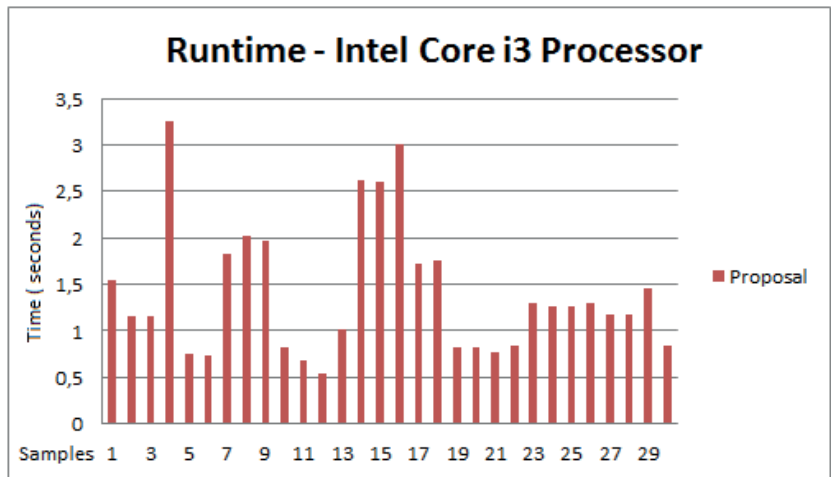

Figure 10 Execution times for the Intel Core i3 processor 


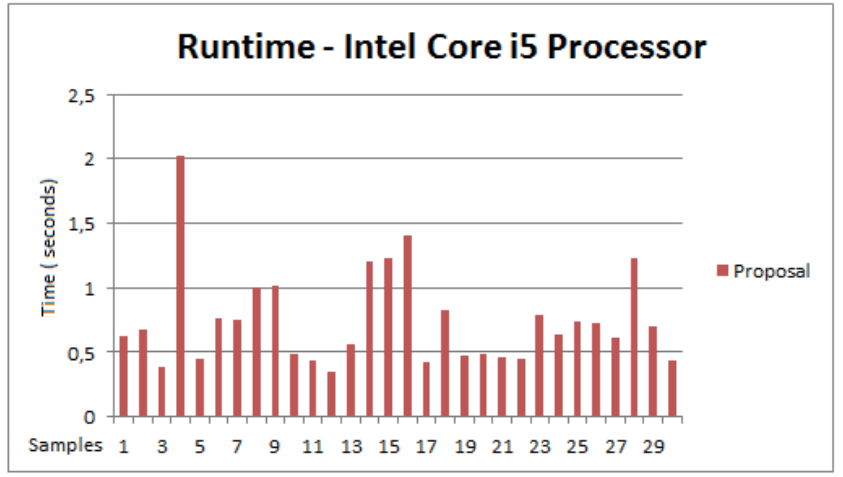

Figure 11 Execution times for the Intel Core i5 processor

An execution time of 3 seconds per sample makes this approach better than the one in [39]. Figure 12 shows the average times to run the proposed algorithm in the Dual Core processor, Intel Core i3 processor, and the Intel Core i5 processor.

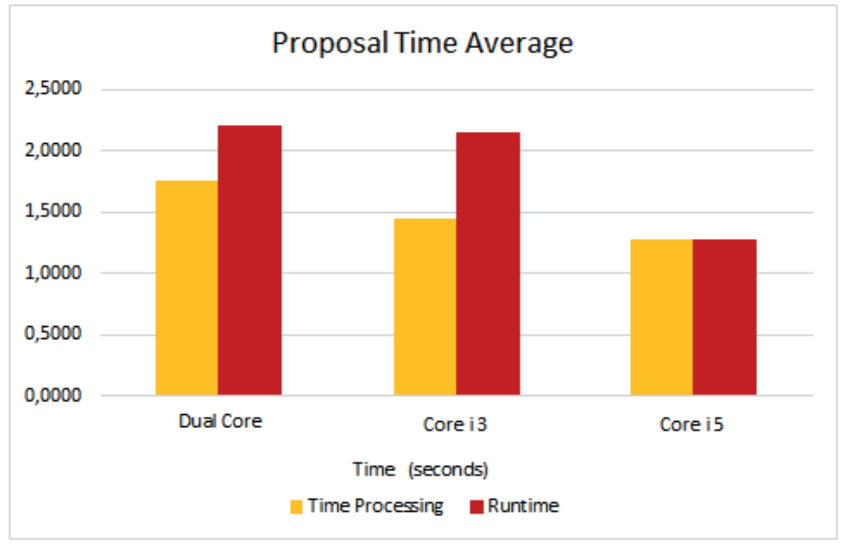

Figure $12 \mathrm{WT}-\mathrm{MO}$ algorithm average time

\section{CONCLUSIONS}

Performing clinical laboratory tests is a distant reality to some healthcare facilities in underdeveloped and developing countries, especially in remote areas. Furthermore, even in developed regions, automated clinical tests can bring more safety to patients and healthcare professionals.

This work introduced the Watershed Transform and Morphological Operations (WT-MOs) algorithm, which delivered results, in all analyzed scenarios and with all samples, with better execution and processing times when compared to other papers. The good performance of the proposed method in different hardware platforms indicates that it is feasible for the different laboratory realities.

The automated confirmation of the diagnosis and the accomplishment of laboratory tests provide higher reliability of the results to both health professionals and patients because the computers reduce the chances of human failures.

The creation of new methodologies, such as the one presented in this paper, results in a considerable reduction in equipment costs without loss in quality and accuracy of hematological diagnoses.

This study is a first step towards the realization of a fully automatic blood cells segmentation and classification subsystem of a computer-aided clinical platform that can deliver telemedical care for remote and hard to reach places [45][46].

In the future, Content-Based Image Retrieval (CBIR) systems can integrate and handle information about blood cells count and fuse it with other image modalities [45][46]. It is worth considering that the development of low-cost mobile microscopic devices can significantly improve the successful deployment of computer vision-based medical diagnosis solutions. Smartphones are now very likely health diagnosis and treatment platforms in poverty-stricken areas.

The image acquisition capabilities and processing power of mobile devices can reduce the effort for exhaustive and time-consuming microscopic examinations. Additionally, less specialized professionals with knowledge about the telemedicine system and preparation of blood smears can supplement the lack of highly trained microscopists in rural areas. The use of mobile devices can also bring important improvements in healthcare portability and biomedical data transmission.

\section{REFERENCES}

[1] K. Barnard, P. Duygulu, N. de Freitas, D. Forsyth, D. Blei, and M.I. Jordan, "Matching words and pictures," JMLR, vol. 3, p. 1107-1135, 2003.

[2] Y. Chen, and J.Z. Wang, "Image categorization by learning and reasoning with regions," $J M L R$, vol. 5, p. 913-939, 2004.

[3] E. A. Mohammed et al. "Chronic lymphocytic leukemia cell segmentation from microscopic blood images using watershed algorithm and optimal thresholding," Proc. 26th IEEE Canadian Conf. of Electrical and Computer Engineering (CCECE), 2013

[4] S.K. Bandyopadhyay, "Method for Blood cell segmentation," Journal of Global Research in Computer Science, 2011.

[5] G.B. Vitor, A. Körbes, R.A. Lotufo, and J.V. Ferreira, "Analysis of a step based watershed algorithm using CUDA, in Nature-Inspired Computing Design, Development, and Applications", Hershey, PA, USA, IGI Global, 2012. doi: 10.4018/978-1-4666-15748.ch018 
[6] F. Meyer, "Watersheds on weighted graphs," Pattern Recognition Letters, vol. 47, 1, pp. 72-79, 2014. doi: 10.1016/j.patrec.2014.02.018.

[7] D. Martin, C. Fowlkes, D. Tal, and J. Malik, "A database of human segmented natural images and its application to evaluating segmentation algorithms and measuring ecological statistics," Proc. 8th Int'l Conf. Computer Vision, vol. 2, p. 416-423, 2001.

[8] J. Shi, and J. Malik, "Motion segmentation and tracking using normalized cuts," Proc. of IEEE 6th Intl. Conf. on Computer Vision, p.1154-60, 1998.

[9] C. Stiller, "Object-based estimation of dense motion fields," IEEE Trans. on Image Processing, vol. 6, no. 2, p. 234-50, 1997.

[10] J. Wang and E. Adelson, "Representing moving images with layers," IEEE Trans. on Image Processing, vol. 3, no. 5, p. 625-638, 1994.

[11] M. Naemura, A. Fukuda, Y. Mizutani, Y. Izumi, Y. Tanaka, and K. Enami, "Morphological segmentation of sports scenes using color information," IEEE Transactions on Broadcasting, vol. 46, 3, 2000.

[12] D. Wang, "Unsupervised video segmentation based on watersheds and temporal tracking," IEEE Trans. on Circuits and Systems for Video Technology, vol. 8, no. 5, p. 539-46, 1998.

[13] G. Halevi, and D. Weinshall, "Motion of disturbances: detection and tracking of multi-body non-rigid motion," Proc. of IEEE Conf. on Computer Vision and Pattern Recognition, pp. 897-902, 1997.

[14] T. Meier, and K.N. Ngan. "Automatic segmentation of moving objects for video object plane generation," IEEE Trans. on Circuits and Systems for Video Technology, vol. 8, no. 5, p. 525-38, 1998.

[15] J. G. Choi, S.W. Lee, and S.D. Kim, "Spatio-temporal video segmentation using a joint similarity measure," IEEE Trans. on Circuits and Systems for Video Technology, vol. 7, no. 2, p. 279-86, 1997.

[16] F. Moscheni, S. Bhattacharjee, and M. Kunt, "Spatiotemporal segmentation based on region merging," IEEE Trans. on Pattern Analysis and Machine Intelligence, vol. 20, no. 9, p. 897-915, 1998.

[17] D. Comaniciu, and P. Meer, "Robust analysis of feature spaces: color image segmentation," Proc. of IEEE Conf. on Comp. Vision and Pattern Recognition, p. 750-755, 1997.

[18] Y. Deng, C. Kenney, M.S. Moore, and B.S. Manjunath, "Peer group filtering and perceptual color image quantization," Proc. of IEEE Intl. Symposium on Circuits and Systems, vol. 4, p. 21- 24, 1999.

[19] S. Beucher, and F. Meyer, "The morphological approach to segmentation: the watershed transformation," in Mathematical Morphology in Image Processing, E. Dougherty (ed.), Marcel Dekker, p. 433-481, 1993.

[20] E.L. Andrade, E. Khan, J.C. Woods, and M. Ghanbari, "Segmentation and tracking using region adjacency graphs, picture trees and prior information," Proc. IEEE Visual Information Engineering (VIE'03), p. 4548, 2003.

[21] L. Jiang, H. Lu, H., V.D. My, A. Koch, and A. Zell, "Superpixel segmentation based gradient maps on RGB-D dataset," Proc. 2015 IEEE Int'l Conference on Robotics and Biomimetics (ROBIO) 2015.
[22] L. C. Junqueira, and J. Carneiro, Biologia Celular e Molecular, São Paulo, Brazil, 9 ${ }^{\text {th }}$ ed., Guanabara Koogan, 2012.

[23] L.C. Junqueira, and J. Carneiro, Histologia Básica, 10th ed., São Paulo: Guanabara, 2004.

[24] A.C.B. Monteiro, D.H. Dorigatti, A.G. Rodrigues, and J.B.M. da Silva, "Sickle cell anemia, a genetic disorder characterized by the change in shape of red blood cells," Saúde em Foco, no 7, 2015.

[25] M. Melo, and C.M. Silveira, Laboratorio de Hematologia: Teorias, Técnicas e Atlas, Rubio, 2015.

[26] A.K. Abbas, A.H. Lichtman, and S. Pillai, Cellular and Molecular Immunology, Amsterdam, The Netherlands, 7th ed., Elsevier, 2012.

[27] R. Hoofman, E.J. Benz Jr., L.E. Silberstein, H. Heslop, J. Weitz, and J. Anastasi, Hematology: Basic Principles and Practice, Canada, 6th ed., Elsevier, 2013.

[28] Abbot Laboratorie de México S.A. Atlas com Interpretacion Histogramas y Escatergramas, Buenos Aires, Argentina, E.G, 2002.

[29] R. Failace, Hemograma. Manual de Interpretação, Porto Alegre, Brazil, $5^{\text {th }}$ ed., Artemed, 2009.

[30] B. Ciesla, Hematology in Practice. 2nd ed., Davis Company, 2012.

[31] M.L. Turgeon, Clinical Hematology Theory, and Procedures. 4th ed. Philadelphia: Lippincott Williams and Wilkins, 2004.

[32] R.C. Gonzalez, R.E. Woods, and S.L. Eddins, Digital Image Processing Using MATLAB. $2^{\mathrm{a}}$ ed. Gatesmarki Publishing, 2009.

[33] A.H. Schmaier, and L.M. Petruzzelli, Hematology for the Medical Student, Lippincott Williams e Wilkins, 2003.

[34] P.H. da Silva, Y. Hashimoto, and H. Bertassoni, Hematologia Laboratorial, Rio de Janeiro, Brazil, Revinter, 2009.

[35] M. Mogra, and V. Srivastava, "A Comprehensive Review of Analysis of Counting Blood Cells Using Different Image Processing Algorithms," International Journal of Eng. Science Invention, ISSN (Online): 2319-6734, ISSN (Print): 2319-6726 www.ijesi.org vol. 3, 6, p. 29-3, 2014.

[36] S.L. Bhagavathi, and S.T. Niba, "An automatic system for detecting and counting $\mathrm{RBC}$ and WBC using Fuzzy Logic," Journal of Engineering and Applied Sciences, 2006-2016 Asian Research Publishing Network (ARPN), vol. 11, no. 11, 2016.

[37] R.C. Gonzalez, R.E. Woods, and S.L. Eddins, Digital Image Processing Using MATLAB. $2^{\mathrm{a}}$ ed. Gatesmarki Publishing, 2009.

[38] R.C. Gonzalez, and R.E. Woods, Digital Image Processing. $3^{\mathrm{a}} \mathrm{ed}$. United States: Pearson: Prentice Hall, 2008.

[39] T. Hemant, S. Sexena, and N. Vadak, "Segmentation using morfological watershed transformation counting blood cells," Int'l J. of Comp, App. \& Inf. Techn., vol. 2, issue III, 2013. 
[40] Mathworks. MATLAB: MATLAB Primer R 2014b. 7 th, 2005.

[41] Mathworks. MATLAB: The Language of Technical Computing, 2011.

[42] N.M. Sobhy, N.M. Salem, and M. Dosoki, "A comparative study of white blood cells segmentation using Otsu threshold and watershed transformation," J. of Biom. Eng. and Med. Im., Society for Science and Education, UK, vol. 3, 3, 2016.

[43] A.M. Coelho, and V.V. Estrela, "EM-based mixture models applied to video event detection," Principal Component Analysis - Engineering Applications, Dr. P. Sanguansat (Ed.), InTech Open, 2012. doi: $10.5772 / 38129$.

[44] S.R. Fernandes, V.V. Estrela, H.A. Magalhaes, and O. Saotome, "On improving sub-pixel accuracy by means of B-spline," Proc. of the 2014 IEEE Int'l Conf. on Im. Syst. and Techn. (IST 2014), Santorini, Greece, 2014. doi: 10.1109/IST.2014.6958448.

[45] M.A. de Jesus, and V.V. Estrela, "An introduction to data mining applied to health-oriented databases," Oriental J. of Comp. Sc. and Techn. (OJCST), vol. 9, 3, p. 177-185, 2016. doi: 10.13005/ojcst/09.03.03.

[46] A.E. Herrmann, and V.V. Estrela, "Content-based image retrieval (CBIR) in remote clinical diagnosis and healthcare," Encyclopedia of E-Health and Telemedicine, Hershey, PA, USA, IGI Global, 2016. doi: 10.4018/978-1-4666-9978-6.Ch039.



Ana Carolina Borges Monteiro. B.Sc. in Biomedicine from Amparense University Center - UNIFIA in 2015. Currently, she is an M.Sc. candidate by Department of Communications, Faculty of Electrical and Computer Engineering at LCV-UNICAMP. She has expertise in

clinical analysis automation (emphasis on hematology), digital image processing, biomedical engineering, and MATLABC software.

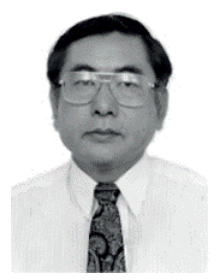

Yuzo Iano . B.Sc. (1972), M.Sc. (1974) and Ph.D. degrees (1986) in Electrical Eng. at UNICAMP, Brazil. Since then he has been working in the technological production field, with 1 patent granted, 8 filed patent applications and 36 projects completed with research and development agencies.

He has supervised 29 doctoral theses, 49 master's dissertations, 74 undergraduate and 48 scientific initiation works. He has participated in 100 master's examination boards, 50 doctoral degrees, author of 2 books and more than 250 published articles. He is currently Professor at UNICAMP, Editor-in-Chief of the SET International Journal of Broadcast Engineering and General Chair of the Brazilian Symposium on Technology (BTSym). He has experience in Electrical Engineering, with knowledge in Telecommunications, Electronics and Information Technology, mainly in the field of audio-visual communications and multimedia.

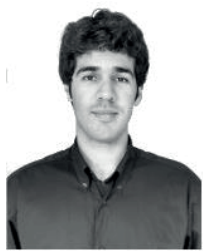

Reinaldo Padilha. B.Sc. in Computer Engineering from the University Regional Center of Espírito Santo de Pinhal in 2014. Currently, he is an M.Sc. degree candidate by Department of Semiconductors, Instruments and Photonics, Faculty of

Electrical and Computer Engineering at the LCVUNICAMP working with technological and scientific research as well as in programming and development in $\mathrm{C} /$ $\mathrm{C}++$, Java and .NET languages. His main topics of interest are simulation, operating systems, software engineering, wireless networks, internet of things, broadcasting and telecommunications systems. 